EPJ Web of Conferences 53, 05001 (2013)

DOI: $10.1051 /$ epjconf/20135305001

(C) Owned by the authors, published by EDP Sciences, 2013

\title{
Search for ultra-high energy photons and neutrinos using Telescope Array surface detector
}

\author{
G.I. Rubtsov ${ }^{1, a}$, M. Fukushima ${ }^{2}$, D. Ivanov ${ }^{3}$, B.T. Stokes ${ }^{3}$, G.B. Thomson ${ }^{4}$ \\ and S.V. Troitsky ${ }^{1}$ for Telescope Array Collaboration ${ }^{b}$ \\ 1 Institute for Nuclear Research of the Russian Academy of Sciences, Moscow, Russia \\ ${ }^{2}$ Institute for Cosmic Ray Research of University of Tokyo, Japan \\ ${ }^{3}$ Rutgers - The State University of New Jersey, Piscataway, USA \\ ${ }^{4}$ University of Utah, High Energy Astrophysics Institute, Salt Lake City, Utah, USA
}

\begin{abstract}
We search for ultra-high energy photons by analyzing geometrical properties of shower fronts of events registered by the Telescope Array surface detector. By making use of an event-by-event statistical method, we derive upper limits on the absolute flux of primary photons with energies above $10^{19} \mathrm{eV}$, $10^{19.5} \mathrm{eV}$ and above $10^{20} \mathrm{eV}$ based on the three years data from Telescope Array surface detector (May 2008 - May 2011). We report the results of down-going neutrino search based on the analysis of very inclined events.
\end{abstract}

\section{INTRODUCTION}

Telescope Array (TA) experiment [1] is a hybrid detector operating in Utah, USA. TA consists of a surface detector array of 507 plastic scintillators with $1.2 \mathrm{~km}$ spacing covering $700 \mathrm{~km}^{2}$ area [2] and three fluorescence detectors [3]. The purpose of this Talk is to present photon and neutrino search capabilities of Telescope Array surface detector and to place the upper limits on the integral photon fluxes in energy region above $10^{19} \mathrm{eV}$.

Several limits on the UHE photon flux have been set by independent experiments, including Haverah Park [4], AGASA [5], Yakutsk [6, 7] (see also reanalyses of the AGASA [8] and AGASA+Yakutsk [9] data at the highest energies) and the Pierre Auger Observatory [10-12], but no evidence for primary photons found at present. Photon limits may be used to constrain the parameters of top-down models [13] and in the future photon search may be used to assess the parameters of astrophysical sources in Greisen-Zatsepin-Kuzmin [14, 15] cut-off scenario which predict photons as everpresent secondaries. Moreover, results of the photon search severely constrain the parameters of Lorentz invariance violation at Planck scale [16-18]. Finally, photons with energies above $\sim 10^{18} \mathrm{eV}$ might be responsible for CR events correlated with BL Lac type objects on the angular scale significantly smaller than the expected deflection of protons in cosmic magnetic fields and thus suggesting neutral primaries $[19,20]$ (see Ref. [21] for a particular mechanism).

Ultra-high energy neutrinos may be generated by the decay of charged pions produced as a secondaries in GZK process [22, 23], by beta-decay of unstable ultra-hugh energy nuclei or by some mechanism related to the new physics. Neutrino flux is constrained by several experiments, see [24] and references therein.

\footnotetext{
ae-mail: grisha@ms2.inr.ac.ru

bFor the full authorlist see Appendix "Collaborations" in this volume

This is an Open Access article distributed under the terms of the Creative Commons Attribution License 2.0, which permits unrestricted use, distribution, and reproduction in any medium, provided the original work is properly cited.
} 


\section{EPJ Web of Conferences}

\section{PHOTON SEARCH}

The Telescope Array surface detector stations contain plastic scintillators of $3 \mathrm{~m}^{2}$ area which detect both muon and electromagnetic components of the extensive air shower and therefore are sensitive to showers induced by primary photons (see e.g. Ref. [25] for discussion). We use the shower front curvature as a composition-sensitive parameter (C-parameter) and we use a modification of event-byevent statistical method [26] to constrain the photon integral flux above the given energy. For the energysensitive parameter (E-parameter), we use the scintillator signal density at $800 \mathrm{~m}$ core distance $\mathcal{S} \equiv S_{800}$.

\subsection{Simulations}

Air showers induced by primary photons differ significantly from the hadron-induced events (see e.g. [27] for a review). At the highest energies there are two competitive effects responsible for the diversity of showers induced by primary photons. First, due to the Landau, Pomeranchuk [28] and Migdal [29] (LPM) effect the electromagnetic cross-section is suppressed at energies $E>10^{19} \mathrm{eV}$. The LPM effect leads to the delay of the first interaction and the shower arrives to the ground level underdeveloped. Another effect is the $e^{ \pm}$pair production due to photon interaction with the geomagnetic field above the atmosphere. Secondary electrons produce gamma rays by synchrotron radiation generating a cascade in the geomagnetic field. The probability of this effect is a function of the square of the product of photon energy and perpendicular component of geomagnetic field. The shower development therefore depends on both zenith and azimuthal angles of photon arrival direction.

Event-by-event method requires us to have a set of simulated photon-induced showers for the analysis of each real shower. We simulate the library of these showers with different primary energies and arrival directions. For the highest energy candidates (events which may be induced by photon with primary energy above $10^{19.5} \mathrm{eV}$ ) we simulate individual sets of showers with fixed zenith and azimuthal angles.

We use CORSIKA [30] with EGS4 [31] model for electromagnetic interactions, PRESHOWER code [32] for geomagnetic interactions, QGSJET II [33] and FLUKA [34] for high and low energy hadronic interactions. There is no significant dependence of the hadronic model because only photoninduced simulated showers are used in calculations. The showers are simulated with thinning and the dethinning procedure is used [35].

Detector response is accounted for by using look-up tables simulated with GEANT4 [36]. Real-time array configuration and detector calibration information are used for each simulated event. Monte-Carlo (MC) events are produced in the same format as real events and analysis procedures are applied in the same way to both. Photon-induced MC set contains $2 \cdot 10^{6}$ triggered events based on 3380 independent CORSIKA showers [37]. No proton simulations are used in calculating the flux limits.

\subsection{Dataset}

We use Telescope Array surface detector dataset covering dates from 2008-05-11 to 2011-05-01. Surface detector has been collecting data for more than $95 \%$ of time during that period [2].

We reconstruct each event with a joint fit of the geometry and lateral distribution function (LDF) and determine Linsley curvature parameter " $a$ " along with the arrival direction, core location and signal density at 800 meters $\mathcal{S} \equiv S_{800}$. The same reconstruction procedure is applied to both data and MonteCarlo events.

For each real event " $i$ " we estimate the energy of hypothetical photon primary $E_{\gamma}^{i}=E_{\gamma}\left(\mathcal{S}^{i}, \theta^{i}, \phi^{i}\right)$, i.e. the average energy of the primary photon, inducing the shower with the same arrival direction and $\mathcal{S}$. The look-up table for $E_{\gamma}(\mathcal{S}, \theta, \phi)$ is built using photon MC set; the dependence on azimuthal angle $\phi$ is relevant for events with $E_{\gamma}>10^{19.5} \mathrm{eV}$ where geomagnetic preshowering is substantial. 

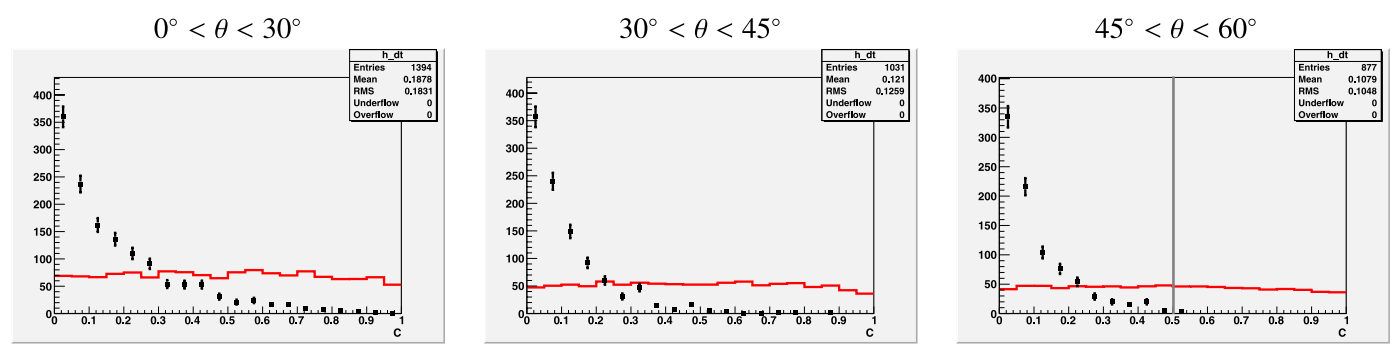

Figure 1. $\mathcal{C}$ distribution for three different zenith angle regions. Black points - data, red line - photon MC with $E^{-2}$ spectrum. Photon median is represented by vertical gray line.

Photon-induced showers are naturally highly fluctuating and consequently the accuracy of determination of $E_{\gamma}$ is $50 \%$ at one sigma level. In the present analysis $E_{\gamma}$ is used for event selection only and therefore it's fluctuations are well accounted in exposure calculation: the result of these fluctuations is a fraction of lost photons [26] which will be estimated in Section 2.4.

The following cuts are applied to both data and MC events:

1. Zenith angle cut: $45^{\circ}<\theta<60^{\circ}$;

2. The number of detectors triggered is 7 or more;

3. Shower core is inside the array boundary with the distance to the boundary larger than 1200 meters;

4. Joint fit quality cut, $\chi^{2} /$ d.o.f. $<5$;

5. $E_{\gamma}\left(S_{800}^{i}, \theta^{i}, \phi^{i}\right)>10^{19} \mathrm{eV}$.

The cuts determine photon registration efficiency which is higher that $50 \%$ for showers induced by primary photons with energy above $10^{19} \mathrm{eV}$. Dataset contains 877 events with $E_{\gamma}>10^{19} \mathrm{eV}$ and $45^{\circ}<\theta<60^{\circ}$.

\subsection{Method}

To estimate the flux limit we use event-by-event method. Linsley curvature parameter " $a$ " is used as a C-observable and $\mathcal{S} \equiv S_{800}$ is used as E-observable. For each real event "i" we estimate the pair of parameters $\left(\mathcal{S}_{o b s}^{i}, a_{o b s}^{i}\right)$ and the arrival direction $\left(\theta^{i}, \phi^{i}\right)$ from the fit of shower front geometry and LDF.

We select a simulated gamma-induced showers compatible with the observed $\theta^{i}, \phi^{i}$ and $\mathcal{S}_{o b s}^{i}$ and calculate the curvature distribution of the simulated showers $f_{\gamma}^{i}(a)$ as discussed in Ref. [26]. For each event, we determine the quantity

$$
\mathcal{C}^{i}=\int_{-\infty}^{a_{\text {obs }}^{i}} f_{\gamma}^{i}(a) d a
$$

which is the value of the integral probability distribution function for the observed curvature. The distribution of $\mathrm{C}$ for data and photon $\mathrm{MC}$ is shown on Fig. 1.

Though the distributions $f_{\gamma}^{i}(a)$ vary with energy and arrival direction, $\mathcal{C}^{i}$ for gamma-ray primaries would be distributed between 0 and 1 uniformly by definition.

Since the simulations of hadron-induced showers depend strongly on the hadronic interaction model, we do not use the hadronic showers simulations in calculation of the limit.

Suppose that the integral flux of primary photons over a given energy range is $F_{\gamma}$. Then we expect to detect

$$
\bar{n}\left(F_{\gamma}\right)=F_{\gamma} A_{\text {geom }}(1-\lambda)
$$




\section{EPJ Web of Conferences}

Table 1. Relative exposure after consecutive application of cuts.

\begin{tabular}{|l|c|c|c|}
\hline & \multicolumn{3}{|c|}{$(1-\lambda)$} \\
Cut & $E_{0}=10^{19}$ & $E_{0}=10^{19.5}$ & $E_{0}=10^{20} \mathrm{eV}$ \\
\hline$n_{\text {det }} \geq 7$ & $72 \%$ & $94 \%$ & $97 \%$ \\
$\chi^{2} /$ d.o.f. $<5$ & $68 \%$ & $89 \%$ & $95 \%$ \\
$\mathcal{S}$ cut & $57 \%$ & $70 \%$ & $95 \%$ \\
\hline Total: & $57 \%$ & $70 \%$ & $95 \%$ \\
\hline
\end{tabular}

photon events in average, where $A_{\text {geom }}$ is the geometrical exposure of the experiment for a given dataset and $\lambda$ is fraction of "lost" photon (i.e. photons with primary energies within the interesting region which failed to enter the dataset due to cuts).

Let $\mathcal{P}(n)$ be a probability to have $n$ photons in a dataset which is defined as a maximum over all subsets of $n$ real events:

$$
\mathcal{P}(n)=\max _{i_{1}<i_{2}<\ldots<i_{n}} \mathcal{P}\left(\left\{i_{1}, \ldots, i_{n}\right\}\right),
$$

where $\mathcal{P}\left(\left\{i_{1}, \ldots, i_{n}\right\}\right)$ is a statistical probability of the subset $\left\{i_{1}, \ldots, i_{n}\right\}$ to be compatible with uniform distribution (i.e. may include $100 \%$ photon events). At this point any non-parametric statistical test may be used to compare the distribution with uniform. We use Smirnov-Cramer-von Mises "omega-square" test because it allows simple and quick numerical procedure to maximize the probability over all subsets of eventset. To constrain the flux $F_{\gamma}$ at the confidence level of $\xi$ one requires

$$
\sum_{n} \mathcal{P}(n) W\left(n, \bar{n}\left(F_{\gamma}\right)\right)<1-\xi
$$

where $W(n, \bar{n})$ is the Poisson distribution with average $\bar{n}$.

The method is conservative by construction and doesn't require any assumptions about hadroninduced showers. This modification of the original method does not require the C-observable to be strongly discriminating (like the muon density used in previous applications $[6,7,9]$ ).

\subsection{Results}

Geometrical exposure for considered SD observation period with $45^{\circ}<\theta<60^{\circ}$ and boundary cut is $A_{\text {geom }}=1286 \mathrm{~km}^{2} \mathrm{sr}$ yr. The fraction of the lost photons is calculated using photon MC set assuming $E^{-2}$ photon spectrum. The values of $(1-\lambda)$ after consecutive application of cuts are given in Table 1.

Using the statistical method (Section 2.3) we arrive to the following results:

$$
\begin{aligned}
& \bar{n}<14.1(95 \% \mathrm{CL}), E_{\gamma}>10^{19} \mathrm{eV}, \\
& \bar{n}<8.7(95 \% \mathrm{CL}), E_{\gamma}>10^{19.5} \mathrm{eV}, \\
& \bar{n}<8.7(95 \% \mathrm{CL}), E_{\gamma}>10^{20} \mathrm{eV} .
\end{aligned}
$$

The constraint on the expected number of photons in the dataset $\bar{n}$ is converted to the flux upper limit with $F_{\gamma}<\bar{n} /\left(A_{\text {geom }}(1-\lambda)\right)$.

$$
\begin{aligned}
& F_{\gamma}<1.9 \cdot 10^{-2} \mathrm{~km}^{-2} \mathrm{sr}^{-1} \mathrm{yr}^{-1}(95 \% \mathrm{CL}), E_{\gamma}>10^{19} \mathrm{eV}, \\
& F_{\gamma}<0.97 \cdot 10^{-2} \mathrm{~km}^{-2} \mathrm{sr}^{-1} \mathrm{yr}^{-1}(95 \% \mathrm{CL}), E_{\gamma}>10^{19.5} \mathrm{eV}, \\
& F_{\gamma}<0.71 \cdot 10^{-2} \mathrm{~km}^{-2} \mathrm{sr}^{-1} \mathrm{yr}^{-1}(95 \% \mathrm{CL}), E_{\gamma}>10^{20} \mathrm{eV} .
\end{aligned}
$$

The above limits along with the results of the other experiments are shown on Fig. 2. 
UHECR 2012

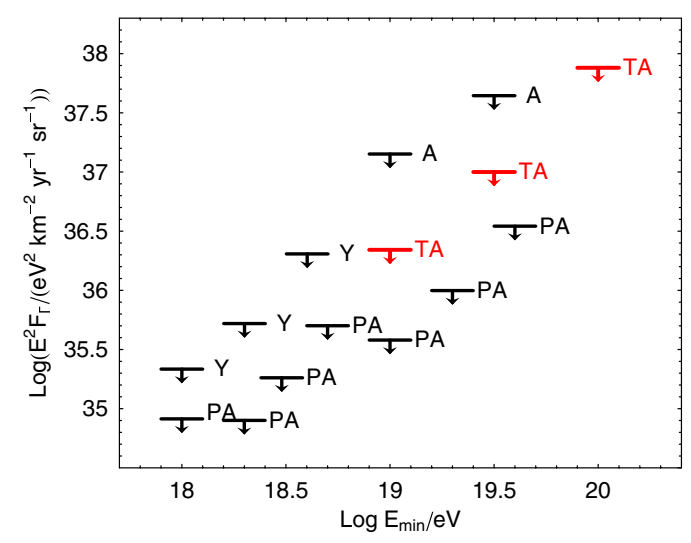

Figure 2. /PRELIMINARY/Photon flux limits of the present work (TA) compared to the limits by AGASA (A) [5], Yakutsk (Y) [7] and Pierre Auger Observatory (PA) [11, 12].

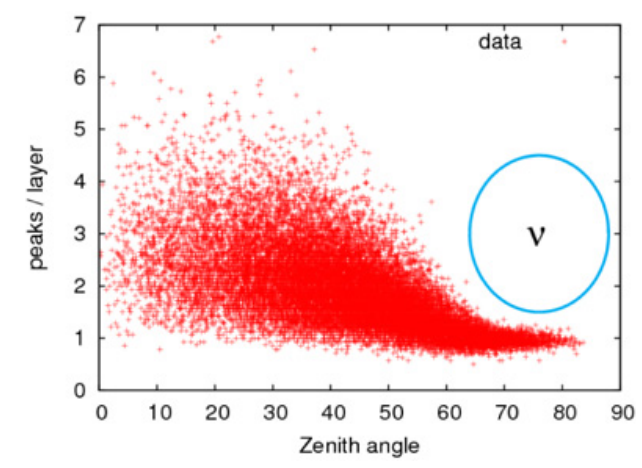

Figure 3. /PRELIMINARY/ Number of peaks per detector layer vs Zenith angle for real events. Circle denotes the area of expected neutrino candidates - very inclined young showers.

We obtain photon fraction limits dividing the corresponding flux limits by the integral flux from the Telescope Array SD spectrum [38]:

$$
\begin{aligned}
& \varepsilon_{\gamma}<6.2 \%(95 \% \mathrm{CL}), E_{\gamma}>10^{19} \mathrm{eV}, \\
& \varepsilon_{\gamma}<28.5 \%(95 \% \mathrm{CL}), E_{\gamma}>10^{19.5} \mathrm{eV} .
\end{aligned}
$$

The result does not depend on the choice of hadronic interaction model, nor on possible systematics in the energy determination of hadronic primaries.

\section{NEUTRINO SEARCH}

As opposed to hadron and photon-induced showers, neutrino-induced shower may be originated in any part of the atmosphere. Therefore very inclined young showers may be considered as a neutrino candidates. To separate young showers we count the number of peaks in the waveforms. To suppress accidental peaks as a result of FADC noise we define a peak as a time bin with signal above 0.2 VEM and higher than a signal of 3 preceeding and 3 consequent time bins. For each event we count the total number of peaks over upper and lower layers of all detectors hit. Very inclined hadronic showers are old and contain mostly muons, which propagate rectilinearly producing mostly single-peaked waveforms. 


\section{EPJ Web of Conferences}

On the contrary, young showers produce long, indented waveforms. Neutrino is expected to produce young showers for all zenith angles.

For neutrino search we use Telescope Array surface detector data from 2008-05-11 to 2011-05-01 with cuts 2, 3 and 4 from Section 2.2. In our dataset, no young showers have zenith angle above $70^{\circ}$, see Fig. 3. Therefore no neutrino candidate events are observed. The calculation of exposure and the neutrnio flux limit is in progress.

The Telescope Array experiment is supported by the Japan Society for the Promotion of Science through Grantsin-Aid for Scientific Research on Specially Promoted Research (21000002) "Extreme Phenomena in the Universe Explored by Highest Energy Cosmic Rays", and the Inter-University Research Program of the Institute for Cosmic Ray Research; by the U.S. National Science Foundation awards PHY-0307098, PHY-0601915, PHY-0703893, PHY-0758342, and PHY-0848320 (Utah) and PHY-0649681 (Rutgers); by the National Research Foundation of Korea (2006-0050031, 2007-0056005, 2007-0093860, 2010-0011378, 2010-0028071, R32-10130); by the Russian Academy of Sciences, RFBR grants 10-02-01406a and 11-02-01528a (INR), by the grants of the President of the Russian Federation MK-1632.2011.2 and NS-5590.2012.2, IISN project No. 4.4509.10 and Belgian Science Policy under IUAP VI/11 (ULB). The foundations of Dr. Ezekiel R. and Edna Wattis Dumke, Willard L. Eccles and the George S. and Dolores Dore Eccles all helped with generous donations. The State of Utah supported the project through its Economic Development Board, and the University of Utah through the Office of the Vice President for Research. The experimental site became available through the cooperation of the Utah School and Institutional Trust Lands Administration (SITLA), U.S. Bureau of Land Management and the U.S. Air Force. We also wish to thank the people and the officials of Millard County, Utah, for their steadfast and warm support. We gratefully acknowledge the contributions from the technical staffs of our home institutions and the University of Utah Center for High Performance Computing (CHPC).

\section{References}

[1] H. Tokuno et al. [Telescope Array Collaboration], J. Phys. Conf. Ser. 293, 012035 (2011)

[2] T. Abu-Zayyad, R. Aida, M. Allen, R. Anderson, R. Azuma, E. Barcikowski, J. W. Belz and D. R. Bergman et al., arXiv:1201.4964. [astro-ph.IM]

[3] H. Tokuno, Y. Tameda, M. Takeda, K. Kadota, D. Ikeda, M. Chikawa, T. Fujii and M. Fukushima et al., Nucl. Instrum. Meth. A 676, 54 (2012)

[4] M. Ave, J. A. Hinton, R. A. Vazquez, A. A. Watson and E. Zas, Phys. Rev. Lett. 85, 2244 (2000)

[5] K. Shinozaki et al., Astrophys. J. 571, L117 (2002)

[6] A. V. Glushkov et al., JETP Lett. 85, 131 (2007)

[7] A. V. Glushkov et al., Phys. Rev. D 82, 041101 (2010)

[8] M. Risse et al., Phys. Rev. Lett. 95, 171102 (2005)

[9] G. I. Rubtsov et al., Phys. Rev. D 73, 063009 (2006)

[10] J. Abraham et al. [Pierre Auger Collaboration], Astropart. Phys. 27, 155 (2007)

[11] J. Abraham et al. [Pierre Auger Collaboration], Astropart. Phys. 29, 243 (2008)

[12] M. Settimo, et al. [The Pierre Auger Collaboration], Proceedings of 32nd ICRC, Beijing, 2011, arXiv:1107.4805

[13] V. Berezinsky, P. Blasi and A. Vilenkin, Phys. Rev. D 58, 103515 (1998)

[14] K. Griesen, Phys. Rev. Lett. 16, 748 (1966)

[15] Z. T. Zatsepin and V. A. Kuz'min, Zh. Eksp. Teor. Fiz. Pis'ma Red. 4, 144 (1966)

[16] S. R. Coleman and S. L. Glashow, Phys. Rev. D 59, 116008 (1999)

[17] M. Galaverni and G. Sigl, Phys. Rev. Lett. 100, 021102 (2008)

[18] A. Saveliev, L. Maccione, G. Sigl, JCAP 1103, 046 (2011)

[19] D. S. Gorbunov et al., JETP Lett. 80, 145 (2004)

[20] R. U. Abbasi et al. [HiRes Collaboration], Astrophys. J. 636, 680 (2006) 


\section{UHECR 2012}

[21] M. Fairbairn, T. Rashba and S. V. Troitsky, Phys. Rev. D 84, 125019 (2011)

[22] V. S. Berezinsky and G. T. Zatsepin, Phys. Lett. B 28, 423 (1969)

[23] O. E. Kalashev, V. A. Kuzmin, D. V. Semikoz et al., Phys. Rev. D 66 (2002) 063004

[24] M. Risse et al., Proceedings of International Symposium on Future Directions in UHECR Physics, (2012)

[25] O. E. Kalashev, G. I. Rubtsov, S. V. Troitsky, Phys. Rev. D 80, 103006 (2009)

[26] D. S. Gorbunov, G. I. Rubtsov and S. V. Troitsky, Astropart. Phys. 28, 28 (2007)

[27] M. Risse and P. Homola, Mod. Phys. Lett. A 22, 749 (2007)

[28] L. D. Landau and I. Ya. Pomeranchuk, Dokl. Acad. Nauk SSSR 92, 535, 735 (1953)

[29] A. B. Migdal, Phys. Rev. 103, 1811 (1956)

[30] D. Heck et al., Report FZKA-6019 (1998), Forschungszentrum Karlsruhe

[31] W. R. Nelson, H. Hirayama, D.W.O. Rogers, SLAC-0265

[32] P. Homola et al., Comp. Phys. Comm. 173 (2005) 71

[33] S. Ostapchenko, Nucl. Phys. Proc. Suppl. 151, 143 (2006)

[34] A. Ferrari et al., CERN-2005-010; A. Fasso, A. Ferrari, J. Ranft et al., eConf C0303241, MOMT005 (2003)

[35] B. T. Stokes, R. Cady, D. Ivanov, J. N. Matthews and G. B. Thomson, Astropart. Phys. 35, 759 (2012)

[36] S. Agostinelli et al. [GEANT4 Collaboration], Nucl. Instrum. Meth. A 506, 250 (2003)

[37] B. Stokes et al., Proc. of the International Symposium on UHECR, Nagoya, December 10-12, (2010)

[38] T.Abu-Zayyad, R. Aida, M. Allen, R. Anderson, R. Azuma, E. Barcikowski, J. W. Belz and D. R. Bergman et al., arXiv:1205.5067 [astro-ph.HE] 